\title{
Parameter-Based Performance Analysis of Object-Based Image Analysis Using Aerial and Quikbird-2 Images
}

\author{
T.Kavzoglu*, M.Yildiz \\ Gebze Institute of Technology, Department of Geodetic and Photogrammetric Engineering, 41400 Gebze Kocaeli, Turkey - \\ (kavzoglu, m.yildiz@gyte.edu.tr)
}

KEY WORDS: Object Based Image Analysis, Segmentation, Nearest Neighbour Classification, VHR Images, Classification Accuracy.

\begin{abstract}
:
Opening new possibilities for research, very high resolution (VHR) imagery acquired by recent commercial satellites and aerial systems requires advanced approaches and techniques that can handle large volume of data with high local variance. Delineation of land use/cover information from VHR images is a hot research topic in remote sensing. In recent years, object-based image analysis (OBIA) has become a popular solution for image analysis tasks as it considers shape, texture and content information associated with the image objects. The most important stage of OBIA is the image segmentation process applied prior to classification. Determination of optimal segmentation parameters is of crucial importance for the performance of the selected classifier. In this study, effectiveness and applicability of the segmentation method in relation to its parameters was analysed using two VHR images, an aerial photo and a Quickbird-2 image. Multi-resolution segmentation technique was employed with its optimal parameters of scale, shape and compactness that were defined after an extensive trail process on the data sets. Nearest neighbour classifier was applied on the segmented images, and then the accuracy assessment was applied. Results show that segmentation parameters have a direct effect on the classification accuracy, and low values of scale-shape combinations produce the highest classification accuracies. Also, compactness parameter was found to be having minimal effect on the construction of image objects, hence it can be set to a constant value in image classification.
\end{abstract}

\section{INTRODUCTION}

Remotely sensed imageries can be effectively used to observe the Earth's surface for constituting land use/cover maps. Recent studies associated with the acquisition of VHR images and development in digital image processing techniques offer a wide range of applications for remote sensing, including environmental monitoring, natural resource management and sustainability of ecosystem services. Since the successful launch of IKONOS in 1999, the number of satellites with high spatial resolution has increased and ground sample distances (i.e. pixel size) of $0.41 \mathrm{~m}$ at panchromatic mode and $1.65 \mathrm{~m}$ at multispectral mode with GeoEye-1 launched in 2008 were reached. Also, WorldView2 launched in 2009 has high spatial resolution with $0.46 \mathrm{~m}$ at pancromatic mode and $1.85 \mathrm{~m}$ at multispectral mode with eight-band. Advances in digital image processing techniques offer a wide range of applications including classification, feature extraction, change detection. Object based image analysis (OBIA), also called object based classification, is a technique used to analyse digital imagery. It has utilized prevalent with improving of image processing techniques over the last decade (Blaschke, 2010). OBIA assesses problems like a human perception and makes evaluations considering several criteria including colour, shape, texture characteristics together with neighbourhoods on real world objects in an image. While pixel based classification considers single pixel contents, object based classification considers information from a set of similar neighbouring pixels called image objects. More specifically, image objects are groups of pixels with similar spectral characteristics.

Although the use of content and texture information has been applied in the last decade, OBIA has demonstrated to be successful and widely used in remote sensing applications (Laliberte and Rango, 2009). OBIA can be considered as a new trend in the classification of satellite images used for Earth observation. Especially, with the increased use of high spatial resolution images, object based classification has become more progressive (Frauman and Wolff, 2005; Kim et al., 2011). Very high spatial resolution images allow to obtain more accurate and detailed mapping of urban land cover; however, its spectral resolution are usually limited with several spectral bands e.g. IKONOS (with 1-m panchromatic and 4-m multispectral resolution) and Quickbird (0.62-m panchromatic and 2.4-m multispectral resolution) images. It has been reported that OBIA offers significant advantages for wide range of studies; including, forest mapping, urban land cover mapping, change detection analysis, agricultural area detection (Chen et al., 2007; Myint et al., 2011; Tzotsos et al., 2011; Klonus et al., 2012).

OBIA is usually applied in three stages: image segmentation, classification and accuracy assessment. Image segmentation is used to merge pixels into objects, and then classification process is implemented based on these objects, instead of individual pixels. Afterwards, an accuracy assessment procedure is applied to test the performance of the classifier. Accuracy assessment is required to compare the land use/cover classification in an image with a reference source or ground information.

One of the advantages of object based classification is that the smallest unit becomes an image object instead of a pixel. In segmentation process, image objects are constituted based on user defined segmentation parameters; scale, compactness and shape. Choices of the segmentation parameters 
significantly affect classification results. Therefore, classification accuracy is directly related to quality of the segmentation process (Meinel and Neubert, 2004; Song et al., 2005; Kim et al., 2009; Marpu et al., 2010). There are many studies in the literature emphasizing the importance of parameter definition in segmentation and their impacts on classification results (e.g. Gao et al., 2007; Lübker and Schaab, 2009; Kim et al., 2011).

The purpose of this research is to determine optimal or nearoptimal segmentation parameters for the data sets; thus infer some practical guidelines for optimal ranges of segmentation parameters. Guidelines for the parameters can be considered as a starting point for a problem similar to the one considered here.

\section{STUDY AREA AND DATA}

Two study sites with different land use/cover features were selected for this study. The first study area is in Kütahya province located between longitudes $38^{\circ} 31^{\prime}$ and $40^{\circ} 30^{\prime}$, between latitudes $40^{\circ} 30^{\prime}$ and $41^{\circ} 30^{\prime}$. For this site, an aerial photo with four spectral bands and $0.3 \mathrm{~m}$ spatial resolution acquired in September 2010 was used. The site is located in south of Kutahya plain covered by farm land, grassland, unmanaged soil and buildings in flat ground. The second study area is in Trabzon province located in north east of Turkey, located between longitudes $38^{\circ} 31^{\prime}$ and $40^{\circ} 30^{\prime}$, between latitudes $40^{\circ} 30^{\prime}$ and $41^{\circ} 30^{\prime}$. A multi-spectral pansharpened Quickbird-2 satellite image having four spectral bands at $0.6 \mathrm{~m}$ spatial resolution acquired in May 2008 was used for the second study site. It has high mountainous terrain covered by forest, farmland and urban classes. For the purpose of evaluation, each of the two images was clipped to a subset of $1500 \times 1500$ pixels (Figure 1). eCognition Developer (v8.7), a widely-used object based image processing software, was applied for all segmentation and classification experiments conducted in this study.

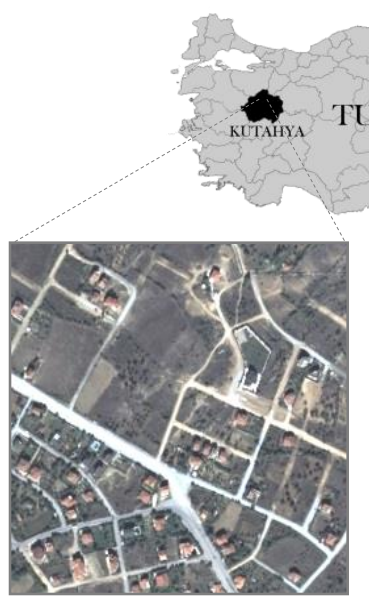

(a)

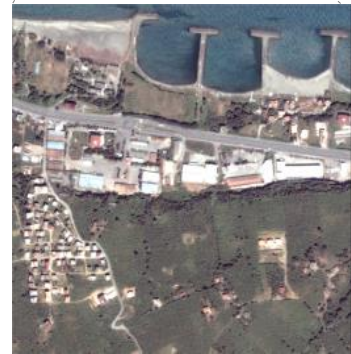

(b)
Figure 1. Location of the study areas, (a) aerial photo of Kutahya and (b) Quickbird-2 image of Trabzon, Turkey.

\section{METHODOLOGY}

\subsection{Image Segmentation}

Segmentation process is the first and crucial step of OBIA creating objects by grouping similar spectral features on the image. It is an important step leading to the analysis of processed image data. Its main goal is to divide an image into meaningful parts that have a strong correlation with objects or areas of the real world contained in the image. In the literature, segmentation methods are traditionally divided into three groups; thresholding (point-based), edge-based and region-based (Blaschke et al., 2004; Sonka et al., 2008). Thresholding is the simplest way of segmentation process that uses a threshold value to convert a gray-scale image into a binary image. In this method, selection of the threshold value directly affects the quality and validity of the results. Edge-based segmentation represents a large group of methods based on information about edges in the image. Although it is one of the earliest segmentation approaches, it is still widely used. Edge-based segmentations rely on edges found in an image using edge detecting operators. These edges mark image locations of discontinuities in gray-level, colour, texture, etc. (Sonka et al., 2008). Region-based techniques, on the other hand, can determine the homogeneity of objects while uncertainties in detecting the exact boundary positions can be reduced by previously extracted edges. Region-based approaches can be also divided into three subgroups as region growing, region merging and splitting/merging techniques. Recent studies have reported methods for the combination of edge and region based method (Mueller et al., 2004).

Multi-resolution segmentation introduced by Baatz and Schäpe (2000) and employed in this research is a bottom-up region merging technique that groups areas of similar and neighbouring pixels into objects by considering homogeneity criteria. As a result, homogeneous areas result in larger objects and heterogeneous areas in smaller ones. Multiresolution segmentation uses three parameters in the process, which are scale, shape and compactness. Scale parameter is considered as the most effective parameter that is used to control the average image object size (Lowe and Guo, 2011; $\mathrm{Li}$ and Shao, 2012). It depends on spatial resolution of the corresponding image and features of the study area. The higher value is selected for scale parameter, the larger the object is obtained. Many researchers have considered the scale parameter as a primary factor for segmentation in object oriented classification studies (Addink et al., 2007; Laliberte and Rango, 2009; Lowe and Guo, 2011; Tzotsos et al., 2011).

As stated by Benz et al. (2004), three scale levels can be applied to images considered for image classification: trees, buildings and roads at a fine scale; groups of trees and groups of buildings aggregated to different settlement types at a medium scale; forest, urban area and open landscape at a coarse scale. Fine scale values allow to form smaller objects than coarse scale values. Image objects created with coarse scales can involve different spectral values because it contains many features of the land cover. Liu and Xia (2010) test the overall effect of segmentation results on object-based classification at different scales, concluding that being the most deterministic parameter scale affects the size of object in general. However, there is neither any certain agreement nor a heuristic in the literature for setting optimum volume for scale parameter. 
Heterogeneity $f$ is the fusion factor defined by $h_{\text {colour }}$ the heterogeneity criterion indicated spectral component, and $h_{\text {shape }}$ the heterogeneity criterion for shape component (Eq. 1). $w_{\text {colour }}$ and $w_{\text {shape }}$ are user-defined weight parameters that allow adjusting the heterogeneity definition. Sum of the weights is equal to 1 (Benz et al., 2004).

$f=w_{\text {colour }} \cdot h_{\text {colour }}+w_{\text {shape }} \cdot h_{\text {shape }}$

$w_{\text {colour }} \& w_{\text {shape }} \in[0,1],\left\{w_{\text {colour }}+w_{\text {shape }}\right\}=1$

$h_{\text {colour }}$ is the spectral component in that, $n_{\text {merge }}$ is the number of pixels within merged object, $n_{o b j 1}$ is the number of pixels in object $1, n_{o b j 2}$ is the number of pixels in object 2 (Eq. 2). In the equation, $c$ is a spectral band index; $w_{c}$ is weight and $\sigma_{c}$ is standard deviation within object of band $c$.

$$
\begin{aligned}
h_{\text {colour }} & =\sum_{c} w_{c}\left(n_{\text {merge }} \cdot \sigma_{c, \text { merge }}\right. \\
& \left.-\left(n_{o b j 1} \cdot \sigma_{c, o b j 1}+n_{o b j 2} \cdot \sigma_{c, o b j 2}\right)\right)
\end{aligned}
$$

$h_{\text {shape }}$ indicates the shape component describing the improvement of the shape concerning smoothness and compactness of an object's shape (Eq. 3). $h_{\text {smooth }}$ and $h_{\text {comp }}$ are shown, where $n$ indicates object size, $l$ is perimeter of object, $b$ is perimeter of object's bounding box. The smoothness heterogeneity equals of the ratio border length $l$ and border length $b$ given by bounding box on an image object, whilst the compactness heterogeneity equals to the ratio border length $l$ and the square root of the number of pixels forming image object (Eq. 4 and 5). The user-defined weight parameters $w_{\text {comp }}$ and $w_{\text {smooth }}$ takes values ranging from 0 to 1 , and sum of these weights is 1 .

$h_{\text {shape }}=w_{\text {comp }} \cdot h_{\text {comp }}+w_{\text {smooth }} \cdot h_{\text {smooth }}$

$$
\begin{aligned}
h_{\text {smooth }} & =n_{\text {merge }} \cdot \frac{l_{\text {merge }}}{b_{\text {merge }}} \\
& -\left(n_{o b j 1} \cdot \frac{l_{o b j 1}}{b_{o b j 1}}+n_{o b j 2} \cdot \frac{l_{o b j 2}}{b_{o b j 2}}\right) \\
h_{\text {comp }}= & n_{\text {merge }} \cdot \frac{l_{\text {merge }}}{\sqrt{n_{\text {merge }}}} \\
& -\left(n_{o b j 1} \cdot \frac{l_{o b j 1}}{\sqrt{n_{o b j 1}}}+n_{o b j 2} \cdot \frac{l_{o b j 2}}{\sqrt{n_{o b j 2}}}\right)
\end{aligned}
$$

The scale parameter computing the limit of $f$ with increasing threshold is the stopping criterion for optimization process. It determines the average size of the segments. Shape parameter provides balance between shape and colour, whilst compactness parameter provides balance between compactness and smoothness. These values are defined by the analyst just before the segmentation process. Trial and error strategy is usually employed in the literature to find the appropriate segmentation parameters for the initial segmentation process (Hofmann et al., 2008; Lowe and Guo, 2011). The values set for the parameters of $w_{\text {shape }}$ and $w_{\text {comp }}$ may significantly affect the form of the resulting objects from a segmented image (Tian and Chen, 2007). In the literature, the weight of compactness parameter was generally set to a fix value of 0.5 by researchers (Myint et al., 2008; Liu and Xia, 2010; Frohn et al., 2011). In this study, compactness parameter was kept constant as 0.5 , scale and shape parameters were varied with different values to analyse the behaviour of classifier performance.

\subsection{Object Based Classification}

Object based image analysis (OBIA or GEOBIA for geospatial object based image analysis) has been widely used in remote sensing applications more than two decades. OBIA has been developed as an alternative to the traditional pixelbased classification. In OBIA, segmented image objects can be classified by two types of classifiers, namely membership functions and the (standard) nearest neighbour classifier, or their combination. Membership functions allow describing the relationship between feature values and the degree of membership to a class using fuzzy logic. It can be defined by the degree of membership, for example any value between one (true) and zero (not true). On the other hand, nearest neighbour classification uses a set of samples of different classes in an attempt to assign class values to a segmented object.

The procedure contains two major steps: firstly teaching the system by giving it certain image objects as samples and secondly classifying image objects in the image object domain based on their nearest sample neighbours (Definiens, 2009). In the application at nearest neighbour classification, samples are selected for training stage similar to supervised classification approach. The training data are usually collected from relatively homogenous sites. The quality and size of the training samples show the degree of representativeness that is crucially important for a successful classification. Accurate and reliable results can be obtained in conjunction with selecting the representative samples to a classification process (Kavzoglu, 2009). The software used in this study utilizes two types of nearest neighbour function, namely standard nearest neighbour and nearest neighbour. The standard nearest neighbour, employed in this study, automatically chooses mean values of objects for all the original bands in the selected image.

\section{RESULTS}

To investigate the effect of segmentation parameters, numerous tests were conducted on the data sets by varying values of segmentation parameters. Afterwards, the quality of segmentation was evaluated according to accuracies of classification. Multi-resolution segmentation algorithm was applied for all segmentation processes conducted in this study. Segmentation parameters, i.e. scale, shape and compactness, were applied to construct image objects. To determine the best appropriate set of parameters, scale, shape and compactness parameters and their effects on classification accuracy were investigated.

\subsection{Parameter Effects in Image Object Construction}

The effects of parameters on the image objects constructed through segmentation were firstly given here to show their impacts in terms of size and shape of image objects. In these experiments, segmentation parameters (i.e. scale, shape and compactness) were varied at certain intervals to obtain image objects. Firstly, to determine the behaviour of scale parameter, segments were created for seven scale values $(5$, $10,20,35,50,70$ ) while holding the shape parameter as 0.1 and compactness parameter as 0.5 . A small portion of the aerial image was presented for more focused and detail view for visual interpretation (Figure 2). It can be easily seen that segments are more distinct in subfigures for scale of 50 and 
70, whilst incoherent segments are apparent in subfigures for scales of 5 and 10. In general, the higher the scale is, the larger the object is obtained. Also, when segment size is increased, it becomes easier to distinguish textural properties of the land use/cover features. After a certain level of scale value, objects start to include multiple land use/cover features. This finding clearly indicates the necessity of optimal value search for the segmentation parameters since both lower and higher values of the parameters introduce some deficiencies into the solution. In other words, segments may include spectral complexity with creating large segments.

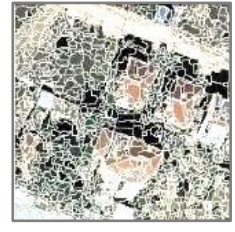

5

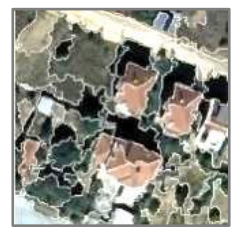

35

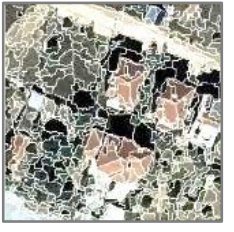

10

50

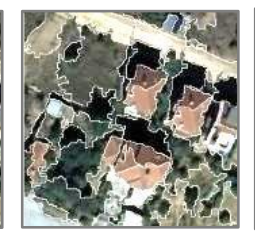

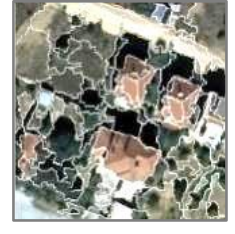

20

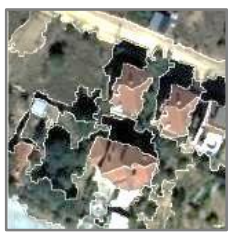

70
Figure 2. Effect of scale parameter on image objects (Shape: 0.1 , Compactness: 0.5).

In order to investigate the effect of shape parameter on image object creation, values were varied between 0.1 and 0.9 with an equal step of 0.2 in that scale was set to 20 and compactness was kept constant as 0.5 for all parameter combinations at this stage (Figure 3 ).

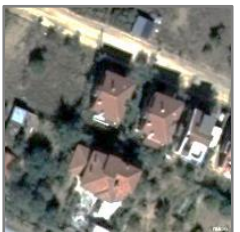

Original

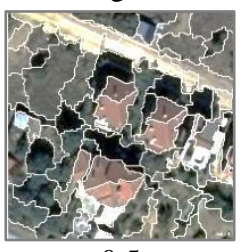

0.5

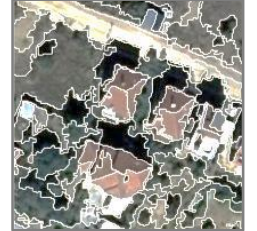

0.1

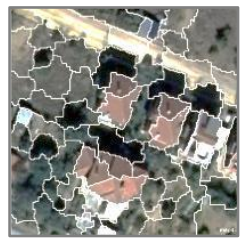

0.7

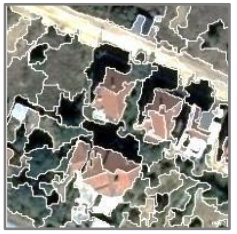

0.3

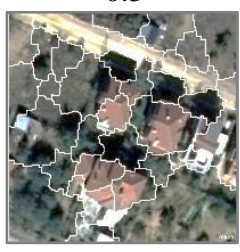

0.9
Figure 3. Effect of shape parameter on image objects (Scale $=20$, Compactness $=0.5$ ).

It was observed that the image objects increased in size without holding the homogenous pixels belonging to the same land use/cover feature, which can significantly reduce the accuracy of subsequent classification. This indicates that the effect of shape parameter is not directly related to the spectral characteristics of the neighbouring pixels. Particularly, when the sub-image created for shape parameter of 0.9 was analysed, mixed image objects of road-soil and tree-soil were produced. As this finding indicates, lower values are advised for shape parameter in segmentation experiments.

In order to observe the effect of compactness parameter on image objects, compactness value was varied between 0.1 and 0.9 whilst keeping the other parameters constant (Figure 4). As the selected object (shadow) shown in red indicates that change in the compactness value did not result in any significant change on the sizes and shapes of the objects. When the other land use/cover features (forest, road and roof shown in yellow, blue and green, respectively) were analysed, slight changes were observed for the shape and size of the image objects. As a result, it was assumed that the compactness parameter did not have considerable effect in the creation of image objects, so compactness values were kept constant as 0.5 for all parameter combinations applied in this study. As a result, main concern of the research became the investigation of optimal scale-shape combination for the data sets considered in this study.

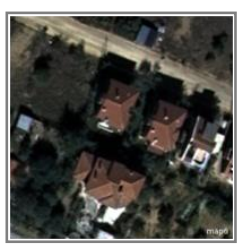

Original

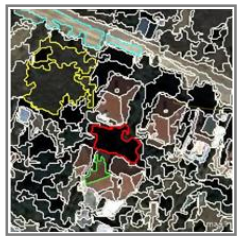

0.5

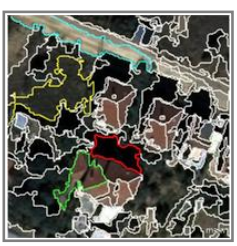

0.1

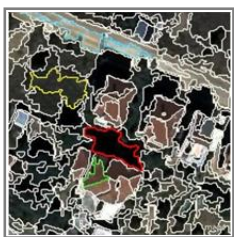

0.7

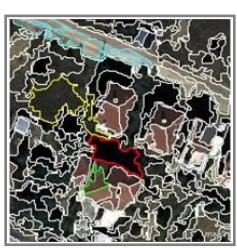

0.3

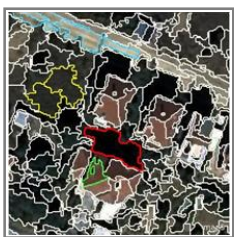

0.9
Figure 4. Effect of compactness parameter on image objects $($ Scale $=20$, Shape $=0.1)$.

\subsection{Effect of Parameter Setting on Classification Accuracy}

Classification accuracy based analysis was performed to evaluate the quality of segmentation results. Segmented images were classified using the standard nearest neighbour classifier, a conventional image classification method. Training data were selected for each class with almost equal numbers to produce more objective accuracy results in this procedure. Training data were collected from each segmented image. Also, segments were enlarged in conjunction with the segmentation parameters.

While the first image was classified into eight land use/cover classes, namely, building, tree, bare soil, pasture, road, earth road, rock and shadow, the second image was classified into seven land use/cover classes, namely, building, water, forest, bare soil, pasture, road and shadow.

Test data sets were created for each study area using the capabilities of the software package. These data sets were then used for the estimation of classification accuracies on resulting images. Variations in classification performances were analysed by comparing overall classification accuracies derived from the error matrix based on TTA (Training and Test Area) mask. Therefore, error matrices were calculated 
separately for each classified images obtained from combinations. Combinations of shape and scale parameters were produced for the datasets. Various scale parameters $(5$, $10,20,35,50$ and 70 ) were chosen while shape parameters were set between 0.1 and 0.9 with an equal step of 0.1 . The overall classification accuracies estimated for all scale-shape combinations are given in Tables 1 and 2. Results for the first dataset show that the highest classification accuracy was produced by $20-0.2$ scale/shape combination $(94.13 \%)$, whilst the lowest accuracy was produced by $70-0.9$ scale/shape combination (48.28\%) (Table 1). For the second study area, the highest classification accuracy was produced by $20-0.1$ scale/shape combination $(88.67 \%)$, whilst the lowest accuracy was produced by $70-0.9$ scale/shape (71.95\%) (Table 2).

\begin{tabular}{|c|c|c|c|c|c|c|}
\hline $\begin{array}{c}\text { Scale } \\
\text { Shape }\end{array}$ & $\mathbf{5}$ & $\mathbf{1 0}$ & $\mathbf{2 0}$ & $\mathbf{3 5}$ & $\mathbf{5 0}$ & $\mathbf{7 0}$ \\
\hline $\mathbf{0 . 1}$ & 89.53 & 89.27 & 90.31 & 92.03 & 85.08 & 80.92 \\
\hline $\mathbf{0 . 2}$ & 90.09 & 92.23 & 94.13 & 87.59 & 85.48 & 80.59 \\
\hline $\mathbf{0 . 3}$ & 91.42 & 92.00 & 90.70 & 89.44 & 80.56 & 79.28 \\
\hline $\mathbf{0 . 4}$ & 89.19 & 90.00 & 87.83 & 82.78 & 78.45 & 76.94 \\
\hline $\mathbf{0 . 5}$ & 89.73 & 92.44 & 88.33 & 81.86 & 82.08 & 74.03 \\
\hline $\mathbf{0 , 6}$ & 90.44 & 88.91 & 86.08 & 84.36 & 79.05 & 72.20 \\
\hline $\mathbf{0 . 7}$ & 90.84 & 87.78 & 83.27 & 84.03 & 75.00 & 65.16 \\
\hline $\mathbf{0 . 8}$ & 86.97 & 86.73 & 85.06 & 71.98 & 69.25 & 59.63 \\
\hline $\mathbf{0 . 9}$ & 85.64 & 84.13 & 72.84 & 64.00 & 54.08 & 48.28 \\
\hline
\end{tabular}

Table 1. Classification accuracies of scale-shape combination for aerial photo. Compactness $=0.5$.

\begin{tabular}{|c|c|c|c|c|c|c|}
\hline $\begin{array}{c}\text { Scale } \\
\text { Shape }\end{array}$ & $\mathbf{5}$ & $\mathbf{1 0}$ & $\mathbf{2 0}$ & $\mathbf{3 5}$ & $\mathbf{5 0}$ & $\mathbf{7 0}$ \\
\hline $\mathbf{0 . 1}$ & 88.22 & 87.96 & 88.67 & 88.56 & 86.30 & 82.34 \\
\hline $\mathbf{0 . 2}$ & 88.43 & 87.87 & 88.33 & 84.94 & 85.72 & 81.96 \\
\hline $\mathbf{0 . 3}$ & 87.61 & 87.59 & 87.91 & 82.02 & 84.35 & 81.82 \\
\hline $\mathbf{0 . 4}$ & 88.29 & 87.68 & 84.79 & 83.28 & 82.07 & 78.69 \\
\hline $\mathbf{0 . 5}$ & 88.30 & 83.75 & 82.65 & 77.66 & 82.39 & 79.79 \\
\hline $\mathbf{0 . 6}$ & 87.27 & 82.91 & 81.67 & 78.11 & 82.29 & 78.24 \\
\hline $\mathbf{0 . 7}$ & 87.69 & 82.26 & 81.05 & 74.76 & 78.89 & 74.55 \\
\hline $\mathbf{0 . 8}$ & 86.26 & 83.13 & 80.40 & 76.25 & 77.12 & 72.77 \\
\hline $\mathbf{0 . 9}$ & 86.55 & 82.83 & 76.31 & 75.35 & 73.88 & 71.95 \\
\hline
\end{tabular}

Table 2. Classification accuracies of scale-shape combination for Quickbird-2 image. Compactness $=0.5$.

Region of upper left-hand corner of tables indicates a specific region (triangular area) producing best performance with high classification accuracy. When the structure of segments on this specific area was analysed, it was found that image comprises over-segments but includes spectral feature of land objects. Optimum segments differ according to characteristics of the study area.

For the first dataset classification accuracies greater than $89.00 \%$ and the second dataset classification accuracies greater than $87.50 \%$ were included in these tables. The main reason for misclassification or low classification accuracy is large segments comprising multiple land use/cover types. Overall, consistently good results were produced for both datasets by the 20-0.2 and 20-0.1 scale-shape combinations, respectively. It was observed that overall accuracies of classifications decreased as the values of scale parameter were increased by greater than 35 .

It was observed that the most appropriate objects to produce accurate results were created by small values of scale and shape parameters. When combinations of shape and scale parameters were formed with small values, image objects were obtained at fine sizes showing real spectral values of the land and classification accuracies, achieving high accurate results consequently. However, when the scale parameter was set to small values (i.e. 5, 10, and 20) and the shape parameter set to high values (i.e. $0.8,0.9$ ), high heterogeneity in segments were observed. In addition, when the scale and shape parameters were set to high values, larger image objects were obtained and lower classification accuracies were obtained. As a consequence, these segments included several land use/cover classes and incoherent segments were created.

Thematic maps were produced using the combinations producing the highest classification accuracy given in Tables 1 and 2 . The first test site was mainly covered by agricultural lands, earth road, bare soil, asphalt road.It should be noted that since the imagery was obtained in the autumn, most of the agricultural lands were identified as soil (Figure 5). A specific class was given to shadow as it is non-geometric events blocking the identification of real-world objects. The second test site mainly has forested lands and residential area (Figure 6). Newly built coastal road with soil and pasture surrounding is apparent in the north part of the image. Also, industrial buildings on the south part of the coastal road were well identified by the classifier. Overall, high accuracy of classification can be easily seen or inferred from clear identification of land use/cover classes in the thematic maps.

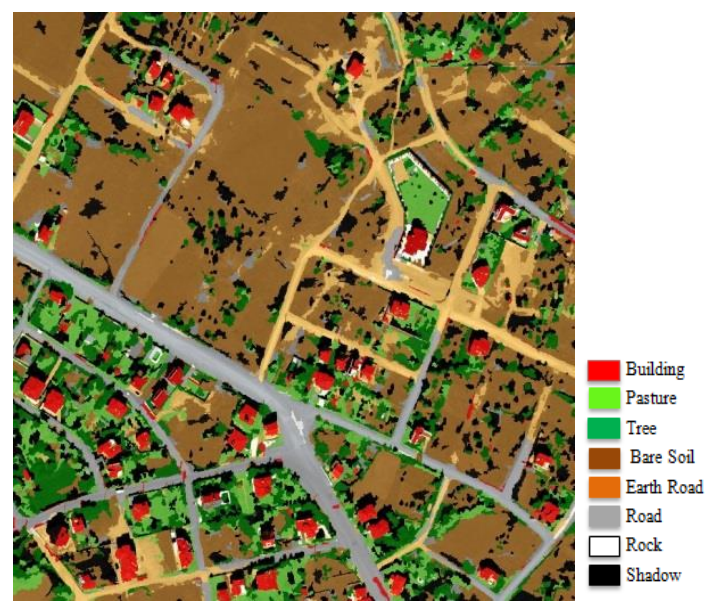

Figure 5. Classification result with optimal parameters for first dataset $($ Scale $=20$, Shape $=0.2$, Compactness $=0.5$ ). 


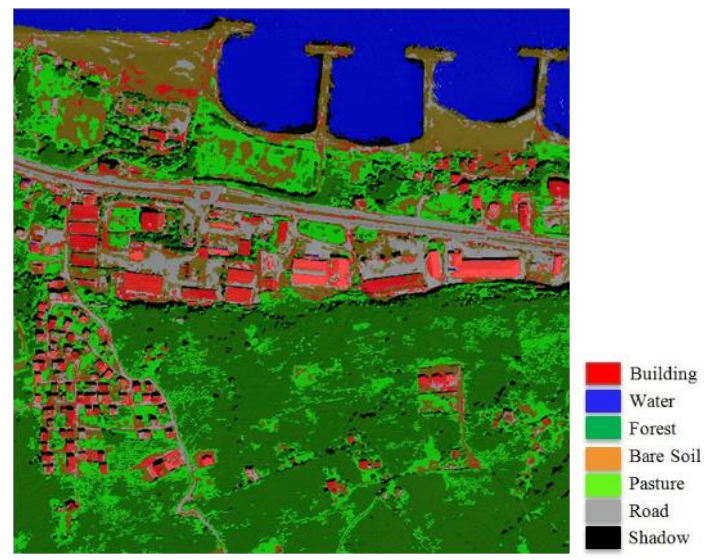

Figure 6. Classification result with optimal parameters for second dataset $($ Scale $=20$, Shape $=0.1$, Compactness $=0.5)$.

\section{CONCLUSIONS}

Setting of optimal values of segmentation is crucially important for a successful classification in object based image analysis. Choosing the segmentation parameters is an essential stage in the image segmentation process, identification ofoptimal segmentation parameters is the subject of a serious research in accordance with the selected imageries and characteristic of study field. The aim of this work was to determinate optimal or near optimal segmentation parameters by heuristics and demonstrate its effect on the classification accuracy using VHR images. Classifications were performed with the nearest-neighbour classifier and the use of representative training data sets is of significant importance for the performance in this process. Training data is directly influenced by the size of image objects obtained through segmentation. It was observed that image objects contained various land use/cover features when larger values of scale and shape were selected.

Classification accuracies obtained through varying parameter settings were presented in tables. Object based classification using aerial photo resulted in $94.13 \%$ overall accuracy with 20-0.2 scale/shape combination. The highest classification accuracy using Quickbird-2 imagery resulted in $88.67 \%$ with $20-0.1$ scale/shape combination. The choice of segmentation parameters can be varied in image segmentation according to the data type, the land cover type, spatial resolution of image and numbers of spectral bands.

Effects of the parameters, particularly scale and shape, were thoroughly analysed by varying their values for both data sets. It should be emphasized that obtained results are valid only for the data sets considered in study area. However, it is believed that they could be valid for similar data sets. Hence, more experiments are needed to confirm the validity of the results. Fast parameter setting is crucial to avoid cumbersome trial-and-error stage during the segmentation process. Experience gained during this study showed that single scaleshape-compactness setting could be insufficient for classifying land use/cover objects since optimal values show variation depending on the size and heterogeneity of the image under consideration.

\section{REFERENCES}

Addink, E.A., De Jong, S.M., Pebesma, E.J., 2007. The importance of scale in object-based mapping of vegetation parameters with hyperspectral imagery. Photogrammetric Engineering and Remote Sensing, 73(8), pp.905-912.

Baatz, M., Schäpe, A., 2000. Multi-resolution segmentationAn optimization approach for high quality multi-scale image segmentation, In: Strobl, J., Blaschke, T., Griesebner, G. (Eds.), Angewandte Geographische Informations Verarbeitung XII. Wichmann-Verlag, Heidelberg, 12-23.

Benz, U.C., Hofmann, P., Willhauck, G., Lingenfelder, I., Heynen, M., 2004. Multi-resolution, object-oriented fuzzy analysis of remote sensing data for GIS-ready information. ISPRS Journal of Photogrammetry and Remote Sensing, 58(3-4), pp.239-258.

Blaschke, T., 2010. Object based image analysis for remote sensing. ISPRS Journal of Photogrammetry and Remote Sensing, 65(1), pp.2-16.

Blaschke, T., Burnett, C., Pekkarinen, A., 2004. Image segmentation methods for object-based analysis and classification. In: Remote Sensing and Digital Image Analysis. Including the Spatial Domain, De Meer, F.and de Jong, S. (Eds.), Kluwer Academic Publishers, Dordrecht, pp. 211-236.

Chen, Y., Shi, P., Fung, T., Wang, J., Li, X., 2007. Objectoriented classification for urban land cover mapping with ASTER imagery. International Journal of Remote Sensing,28(20), pp.4645-4651.

Definiens. 2009. eCognition Developer 8. User Guide. Munich, Germany, Definiens AG.

Frauman, E., E. Wolff., 2005. Segmentation of very high spatial resolution satellite images in urban areas for segments-based classification. Proceedings for 3rd International Symposium Remote Sensing and Data Fusion Over Urban Areas. Tempe, Arizona, USA.

Frohn, R. C., Autrey, B. C., Lane, C. R., Reif, M., 2011. Segmentation and object-oriented classification of wetlands in a karst Florida landscape using multi-season Landsat-7 ETM+ imagery. International Journal of Remote Sensing, 32(5), pp.1471-1489.

Gao, Y., Kerle, N., Mas, J.F., Navarrete, A., Niemeyer, I., 2007. Optimised image segmentation and its effect on classification accuracy. In: Proceedings of the 5th International Symposium on Spatial Data Quality (ISSDQ 2007), Enschede, Netherlands.

Hofmann, P., Strobl, J., Blaschke, T., Kux, H., 2008. Detecting informal settlements from Quickbird data in Rio de Janeiro using an object based approach. In: Object-Based Image Analysis, Blaschke, T., Hay, G., and Lang, S. (Eds.), pp.531-553, Berlin, Springer.

Kavzoglu, T., 2009. Increasing the accuracy of neural network classification using refined training data. Environmental Modelling and Software, 24(7), pp.850-858.

Kim, M., Madden, M., Warner, T.A., 2009. Forest type mapping using object-specific texture measures from multispectral IKONOS imagery: Segmentation quality and image classification issues. Photogrammetric Engineering and Remote Sensing, 75(7), pp.819-829. 
Kim, M., Warner, T. A., Madden, M., Atkinson, D. S., 2011. Multi-scale GEOBIA with very high spatial resolution digital aerial imagery: Scale, texture and image objects. International Journal of Remote Sensing, 32(10), pp.28252850 .

Klonus, S., Tomowski, D., Ehlers, M., Reinartz, P., Michel, U., 2012. Combined edge segment texture analysis for the detection of damaged buildings in crisis areas. IEEE Journal of Selected Topics in Applied Earth Observations and Remote Sensing, 5(4), pp.1118-1128.

Laliberte, A.S., Rango, A. 2009. Texture and scale in objectbased analysis of subdecimeter resolution unmanned aerial vehicle (UAV) imagery. IEEE Transactions on Geoscience and Remote Sensing, 47(3), pp.761-770.

Li, C., Shao, G., 2012. Object-oriented classification of land use/cover using digital aerial orthophotography. International Journal of Remote Sensing, 33(4), pp.922-938.

Liu, D., Xia, F., 2010. Assessing object-based classification: Advantages and limitations. Remote Sensing Letters, 1(4), pp.187-194.

Lowe, S.H., Guo, X., 2011. Detecting an Optimal Scale Parameter in Object-Oriented Classification. IEEE Journal of Selected Topics in Applied Earth Observations and Remote Sensing, 4(4), pp.890-895.

Lübker, T., Schaab, G., 2009. Optimization of parameter settings for multilevel image segmentation in GEOBIA., Proceedings of ISPRS Hannover Workshop, High-Resolution Earth Imaging for Geospatial Information (digital), Hannover, Germany, 2-5 June 2009.

Marpu, P. R., Neubert, M., Herold, H., Niemeyer, I., 2010. Enhanced evaluation of image segmentation results. Journal of Spatial Science, 55(1), pp.55-68.

Meinel, G., and Neubert, M., 2004. A comparison of segmentation programs for high resolution remote sensing data. International Archives of Photogrammetry and Remote Sensing, 35(Part B), pp.1097-1105.

Mueller, M., Segl, K., Kaufmann, H., 2004. Edge-and regionbased segmentation technique for the extraction of large, man-made objects in high-resolution satellite imagery. Pattern recognition, 37(8), pp.1619-1628.

Myint, S. W., Yuan, M., Cerveny, R. S., Giri, C. P., 2008. Comparison of remote sensing image processing techniques to identify tornado damage areas from Landsat TM data. Sensors, 8(2), pp.1128-1156.

Myint, S. W., Gober, P., Brazel, A., Grossman-Clarke, S., Weng, Q., 2011. Per-pixel vs. object-based classification of urban land cover extraction using high spatial resolution imagery. Remote Sensing of Environment, 115(5), pp.11451161.

Song, M., Civco, D. L., Hurd, J. D., 2005. A competitive pixel-object approach for land cover classification. International Journal of Remote Sensing, 26(22), pp.49814997.

Sonka, M., Hlavac, V., Boyle, R., 2008. Image Processing, Analysis, and Machine Vision, International Student Edition, Thomson Learning, 3th edition, pp.175-237.

Tian, J., Chen, D. M., 2007. Optimization in multi-scale segmentation of high-resolution satellite images for artificial feature recognition. International Journal of Remote Sensing, 28(20), pp.4625-4644.

Tzotsos, A., Karantzalos, K., Argialas, D., 2011. Objectbased image analysis through nonlinear scale-space filtering. ISPRS Journal of Photogrammetry and Remote Sensing, 66(1), pp.2-16. 